\title{
Comparative analysis of diagnostic accuracy of different brain biopsy procedures
}

\author{
Deepali Jain, Mehar Chand Sharma, Chitra Sarkar, Deepak Gupta*, Manmohan Singh*, \\ A. K. Mahapatra* \\ Departments of Pathology and *Neurosurgery, All India Institute of Medical Sciences, New Delhi, India
}

Background: Image-guided procedures such as computed tomography (CT) guided, neuronavigator-guided and ultrasound-guided methods can assist neurosurgeons in localizing the intraparenchymal lesion of the brain. However, despite improvements in the imaging techniques, an accurate diagnosis of intrinsic lesion requires tissue sampling and histological verification. Aims: The present study was carried out to examine the reliability of the diagnoses made on tumor sample obtained via different stereotactic and ultrasound-guided brain biopsy procedures. Materials and Methods: A retrospective analysis was conducted of all brain biopsies (frame-based and frameless stereotactic and ultrasound-guided) performed in a single tertiary care neurosciences center between 1995 and 2005. The overall diagnostic accuracy achieved on histopathology and correlation with type of biopsy technique was evaluated. Results: A total of 130 cases were included, which consisted of 82 males and 48 females. Age ranged from 4 to 75 years (mean age 39.5 years). Twenty per cent (27 patients) were in the pediatric age group, while $12 \%$ (16 patients) were $\geq 60$-years of age. A definitive histological diagnosis was established in 109 cases (diagnostic yield 80.2\%), which encompassed 101 neoplastic and eight nonneoplastic lesions. Framebased, frameless stereotactic and ultrasound-guided biopsies were done in 95, 15 and 20 patients respectively. Although the numbers of cases were small there was trend for better yield with frameless image-guided stereotactic biopsy and maximum diagnostic yield was obtained i.e., $87 \%(13 / 15)$ in comparison to conventional frame-based CT-guided stereotactic biopsy and ultrasound-guided biopsy. Conclusions: Overall, a trend of higher diagnostic yield was seen in cases with frameless image-guided stereotactic biopsy. Thus, this small series confirms that frameless neuronavigator-guided stereotactic procedures represent the lesion sufficiently in order to make histopathologic diagnosis.
Key words: Diagnostic yield, frame-based, frameless, histopathology, stereotactic biopsy, ultrasound-guided

Recently, there has been an increasing trend in most branches of surgery towards a reduction in the morbidity of a procedure by limiting surgical entry. This usually involves the application of technology such as laparoscopic cholecystectomy. With regard to brain lesion diagnosis and surgery, technological advances include the use of computer-assisted stereotaxis, intraoperative ultrasound, magnetic resonance imaging, brain mapping and endoscopy.

\section{Computer-assisted stereotactic biopsy}

Stereotaxis refers to a system of navigating to any point within the brain with the aid of imaging techniques that concurrently display external reference landmarks and neural structures. This is a routine procedure in many centers to achieve a histological diagnosis in brain tumors not accessible to open surgery. ${ }^{[1]}$ The advantage of the stereotactic system lies in its ability to allow smaller exposures and to navigate, with minimal disruption of overlying brain, to reach a brain tumor. The earliest stereotactic systems were based on information obtained from ventriculograms and were used mainly for the treatment of Parkinson's disease. ${ }^{[2]}$ The next generation of stereotactic systems used computed tomography (CT) or magnetic resonance imaging (MRI) guidance for localization and a small computer for calculations. This was useful for biopsy purposes and for stereotactic craniotomies. ${ }^{[3]}$ Currently neuronavigation is carried out without requiring external reference landmarks (frame).

\section{Frame-based stereotaxis}

An external head frame is used during 1 brain imaging and this provides reference points from which a computer can calculate and present trajectories and depths to any target point within the 
image of the brain. The main limitations for the application of stereotactic apparatuses are related to the devices' point-based, single trajectory nature and the cost of using stereotactic frames. Furthermore, their use requires time-consuming calculations, which are cumbersome for the surgeon and inconvenient for the patient. $^{[4]}$

\section{Frameless stereotaxis (Image-guided stereotaxis)}

Brain biopsy procedures in which guidance is provided by a frameless stereotactic surgical navigation system with scalpapplied fiducial markers. Several self-adhesive skin markers are applied to the patient's head to direct the probe into the brain. This procedure, called "frameless," or computer-assisted imageguided brain needle biopsy, is very comfortable for the patient and provides the same accuracy as that of the rigid frame. In this, computers can navigate the operator through 3D coordinates and thus fulfill the need for enhanced visibility during surgical procedures. Advantages of image-guided stereotaxis are related to its less invasiveness, better tolerance by the patient and accurate diagnosis. $^{[5]}$

\section{Ultrasound-guided biopsy}

Despite the superior accuracy of stereotactic biopsy, experience demonstrates that ultrasound guidance is feasible and has certain advantages. The ultrasound method for brain biopsy is found to be simple, quick and cost-effective. A significant advantage of the ultrasound is that it provides real-time information of a brain lesion; shift in tumor position or postbiopsy complications can be visualized promptly in the operating theater. However its anatomical resolution is inferior to $\mathrm{CT}$ and is suitable only for lesions larger than $5 \mathrm{~mm}$ in diameter: ${ }^{[6,7]}$

Although numerous studies have been published with regard to the overall diagnostic accuracy of stereotactic brain biopsies only a few have compared the diagnostic efficacy of different types of biopsy procedures. In this retrospective study we describe our experience with 130 brain biopsies and compare the diagnostic yield of different biopsy procedures.

\section{Materials and Methods}

Using CT and/or MRI and ultrasonography (USG), 130 cases of intra-axial brain mass lesions underwent brain biopsy procedure. These biopsies were performed over a period of 11 years (January 1995 to December 2005) at our institute. Biopsies included 95 frame-based CT-guided stereotactic, 20 ultrasoundguided and 15 frameless image-guided stereotactic brain biopsies. These biopsies were undertaken for deep-seated, multiple lesions and the lesions of eloquent areas. CT-guided and ultrasound (US)guided biopsies were done under local anesthesia while imageguided biopsies were performed under general anesthesia. Type of biopsy procedure was not based on the size of the lesion, age of the patient and neurological status of the patient.

\section{Frame-based CT-guided stereotactic biopsy}

Performed with the CT-compatible head frame of the Leksell stereotactic apparatus model-D and Backlund biopsy kit in conjunction with a Picker USA 1991 (PQ-2000) model CT scanner. Following frame fixation in the operation theatre, the patient was shifted to the $\mathrm{CT}$ console, where coordinates were calculated. The patient was then shifted back to the operation theatre. A burr hole was made over the appropriate place and biopsy performed. The burr hole site and the trajectory was chosen according to the site of the lesion.

\section{Frameless Image-guided biopsy}

The image-guided system i.e. stealth station treatment guidance platform (Treon, Sofamor-Danek, Medtronic Inc, Memphis, TN, USA) was used to perform the biopsy. A burr hole was made at the proposed entry site and biopsy needle was advanced under image guidance into the lesion and multiple biopsies were obtained.

\section{US-guided biopsy}

US-guided biopsies were performed using a burr hole, over an appropriate area, through a linear scalp incision and were performed using a real time Panther Ultrasound Scanner 2002 (Advanced Diagnostic Imaging, B and K, Medical) with $6.5 \mathrm{MHz}$ transducer. USG probe was fixed to allow rigid fixation. The biopsy clamp was then attached to the USG probe and the cannula with stylet in place was first passed along the trajectory under ultrasonic visualization. Once the target area was reached, the stylet was removed from the cannula and the biopsy forceps was passed. Multiple biopsies were obtained with continuous real time monitoring. Once the adequate number of tissue samples was obtained, the cannula was removed and a continuous monitoring of the biopsied area was performed with the sonic probe for at least $15 \mathrm{~min}$ to look for any hematoma formation. Following this, the wound was closed. A noncontrast CT was performed within six to eight hours after the procedure to rule out any hematoma formation.

\section{Histopathological evaluation}

Biopsies were fixed in 10\% neutral buffered formalin, routinely processed and paraffin embedded. Five micron thick sections were stained by hematoxylin and eosin (H\&E) stain. Three histopathologists (DJ, MCS, CS) independently reviewed the H\&E slides along with relevant immunohistochemical / special stains and reconfirmed the original histopathological diagnosis. Tumors were diagnosed as per the criteria of WHO classification of CNS tumors (2000).

\section{Immunohistochemistry}

Representative blocks of formalin-fixed paraffin-embedded tissue were selected, five micron thick sections cut and immunohistochemical (IHC) staining performed by LSAB technique using monoclonal antibodies to glial fibrillary acidic 
protein (GFAP; dil1:800), leukocyte common antigen (LCA; dil 1:100), CD3 (dil 1:200), CD20 (dil 1:200,) and cytokeratin (CK; dil 1:25). All antibodies were obtained from M/s Dakopatts, Denmark. High temperature antigen retrieval using microwave was carried out wherever required.

\section{Special stains}

Ziehl Neelsen stain for acid-fast bacilli, periodic acid Schiff (PAS) and silver methenamine stain for fungus were done in all cases with granulomatous inflammation.

\section{Results}

A total of 130 biopsies were done during this study period of 11 years (between January 1995 and December 2005).

\section{Clinical profile \\ Age and sex ratio}

The age ranged from 4 to 75 years (mean 39.5 years). Most of the patients were in their third and fourth decades of life $(42.3 \%)$, while 27 patients $(20.76 \%)$ were in the pediatric age group $(\leq 18$ years) and 16 patients $(12.3 \%)$ were $\leq 60$ years of age. There was male preponderance (82 cases) with male to female ratio of 1.7:1.

\section{Histopathology}

A definitive diagnosis could be offered in 109 (83.84\%) cases which consisted of 101 neoplastic and eight nonneoplastic lesions [Table 1]. Diagnostic yield of individual biopsy technique is given in Table 1.

\section{Frame-based Stereotactic biopsy}

Conventional frame-based CT-guided stereotactic biopsies were performed in 95 patients. The diagnostic accuracy of histological diagnoses was $84.21 \%$ (80/95). In $15.78 \%$ of the cases (15/95), a definitive diagnosis could not be made. Astrocytic neoplasms were the commonest which included nine low grade astrocytomas, 38 diffuse astrocytomas, 11 anaplastic astrocytomas and five glioblastoma multiforme [Table 2]. Of the 63 astrocytic tumors 35 were located in the thalamus, 15 were hemispheric (eloquent areas), four in the corpus callosum, five in brainstem, one multiple, one in basal ganglia and two in third ventricle region. All the five cases of primary central nervous system lymphomas were B immunophenotype. One patient had metastatic adenocarcinoma, with unknown primary.

Of the 18 cases where definite diagnosis was not made, 10 biopsies included only normal brain tissue and in four biopsies there was no evidence of tumor and out of these one showed reactive gliosis. One biopsy was inadequate for opinion as size was very small (less than $0.1 \mathrm{~cm}$ ) [Table 3].

\section{Frameless Image-guided biopsy}

Fifteen patients underwent image-guided brain biopsy. The tumor location among these 15 patients was hemispheric in eight and thalamic in seven. The biopsy specimens showed conclusive
Table 1: Diagnostic yield of different biopsy procedures

\begin{tabular}{lccc}
\hline & $\begin{array}{c}\text { Frame-based } \\
\text { stereotactic } \\
\text { biopsy }\end{array}$ & $\begin{array}{c}\text { Ultrasound- } \\
\text { guided } \\
\text { biopsy }\end{array}$ & $\begin{array}{c}\text { Frameless } \\
\text { Image-guided } \\
\text { biopsy }\end{array}$ \\
No. of biopsies & 95 & 20 & 15 \\
Conclusive diagnosis & 80 & 16 & 13 \\
Diagnostic yield (\%) & 84.21 & 80 & 87 \\
\hline
\end{tabular}

$P$ value 0.85 (not significant)

Table 2: Cases with definite histological diagnosis $(n=109)$

Histological Frame-based Frameless Ultrasounddiagnosis stereotactic Image-guided guided

biopsy biopsy biopsy

Astrocytoma -

09

02

05

WHO Grade I

Diffuse astrocytoma -

38

02

03

WHO Grade II

Anaplastic astrocytoma - 11

WHO Grade III

Glioblastoma multiforme - 05

03

04

WHO Grade IV

05

02

Mixed glioma

02

Oligodendroglioma

Central neurocytoma

Lymphoma

01

01

05

Adenocarcinoma

metastatic

Tuberculosis

Abscess

01

Mucormycosis

03

01

Nonspecific inflammatory 03

pathology

Table 3: Cases with inconclusive diagnoses $(n=21)$

\begin{tabular}{lccc} 
Diagnoses & $\begin{array}{c}\text { Frame-based } \\
\text { stereotactic } \\
\text { biopsy }\end{array}$ & $\begin{array}{c}\text { Frameless } \\
\text { Image-guided } \\
\text { biopsy }\end{array}$ & $\begin{array}{c}\text { Ultrasound- } \\
\text { guided } \\
\text { biopsy }\end{array}$ \\
Normal brain & 10 & - & 01 \\
Gliosis, no tumor & 04 & - & - \\
Inadequate for opinion & 01 & 01 & 02 \\
Fibrocollagenous tissue & - & - & 01 \\
Necrotic tissue & - & 01 & - \\
\hline
\end{tabular}

diagnosis in 13 patients ( $87 \%$ ). In one patient the biopsy showed necrotic material and in one other patient it was inconclusive due to small size of the biopsy [Tables 2 and 3].

\section{Ultrasound-guided biopsy}

US-guided biopsies were done in 20 cases. Of these, 16 had conclusive diagnosis (80\%) for tumor pathology. Among the four nondiagnostic biopsies, two biopsies were too tiny for any diagnosis, one included normal brain and one other biopsy included fibrocollagenous tissue only. Ten thalamic, eight hemispheric and one case each of posterior fossa and suprasellar region were targeted for US-guided biopsy. There were 14 astrocytomas and one case each of NHL B cell type and mucormycosis [Tables 2 and 3].

\section{Discussion}

Literature on comparison of the diagnostic yield of different brain biopsy techniques is sparse. Hence this study was undertaken 
to evaluate diagnostic yield of conventional CT-guided stereotactic, modern real-time frameless image guided stereotactic, and ultrasound-guided biopsy procedures. Diagnostic accuracy of only paraffin-embedded section is analyzed but cytological or frozen section diagnoses were not compared in the present study. Technically, CT allows early recognition and accurate definition of cerebral lesions whereas open intraoperative near real time imaging transforms a blind conventional stereotactic procedure into a visually controlled one. ${ }^{[8]}$

Stereotactic biopsy for intracranial lesions is a reliable and relatively safe procedure and is also a very efficacious method especially in patients who need histological confirmation for the treatment. The overall diagnostic accuracy varies from 80 to $99 \%{ }^{[9]}$ Because of the small sample size of specimens, communication with the neurosurgeon as well as correlation with clinical and radiographic information is necessary to increase the diagnostic yield. However, in order to derive maximum benefit from the tissue obtained by stereotactic biopsy, diagnosis needs to be rapid and accurate. Processing of frozen sections and cytology smears is rapid and generally preferred over permanent sections, which are more time-consuming. These intraoperative diagnostic techniques ultimately help not only in confirming if the target lesion has been reached, but also help in making a sufficiently reliable diagnosis. ${ }^{[10,11]}$

Cytological evaluations are generally in the form of smears and squash preparations. The overall diagnostic yield of cytology varies between 73 and 94\%. ${ }^{[12-14]}$ Firlik et al studied 595 cases and observed correlation of cytology with final diagnosis in $90 \%$ cases (52\% complete and $38 \%$ partial correlation $)^{[15]}$ but Collaco et al reported slightly lower $(73 \%)$ correlation of solid lesions. ${ }^{[16]}$ In the presence of typical morphologic features cytological analysis of tumors can be rapid and reliable, while it often lacks in identifying the grade of neoplasms.

Frozen section evaluation is generally preferred over cytology for intraoperative evaluation of larger biopsy samples. Grunert et $a l^{[17]}$ reported a diagnostic yield of $98 \%$, in intraoperative frozen section and conventional paraffin-embedd sections. However, routine processing of small biopsies helps in the preservation of morphology and permits better assessment.

Several studies have examined the diagnostic accuracy of the stereotactic biopsy procedures by comparing the biopsy diagnosis with the diagnosis of subsequent specimen obtained by craniotomy. Good correlation has been obtained in most of the studies, however; some studies have reported lower accuracy rates. ${ }^{[18]}$ Bernays et $a l^{[19]}$ offered a specific histological diagnosis in 111 of 114 tissues $(97.4 \%)$, which is in contrast with a $79 \%$ accuracy reported by McGirt et $a l,{ }^{[20]}$ evaluating 23 patients who underwent an open surgery within 60 days of stereotactic biopsy. However, in a recent study by Aker et $a l^{[21]}$ the overall diagnostic yield of $94 \%$ reduced to $83 \%$, when correlated with the diagnoses of the resected specimens. The reasons for low accuracy rates may be related either to surgical procedure itself or due to certain inherent characteristics of small brain tumor biopsy specimens. ${ }^{[22]}$ For instance, presence of nonneoplastic reactive tissue elements may obscure nearby or underlying tumor, and lead to a mistaken alternate diagnosis.

The entire procedure is time-consuming and costly. In addition, stereotactic frames are uncomfortable for patients and may impede intraoperative neurological evaluation. ${ }^{[23]}$

Targeting for functional stereotactic procedures is traditionally carried out in a noninteractive fashion without real-time positional feedback. ${ }^{[24]}$ As the availability of image-guided surgical navigation systems has increased, the application of frame-based stereotactic biopsy has declined. Intraoperative imaging allows excellent target localization and many advantages over the conventional frame-based stereotactic biopsy procedure. Immediate postoperative imaging in the operating room allows assessment of adverse events and the potential for immediate management of hemorrhagic complications. ${ }^{[25]}$

Image-guided stereotactic brain biopsy is considered an accurate and safe procedure. ${ }^{[26]}$ A meta analysis of 7471 reported cases demonstrated that a positive histological diagnosis can be obtained by performing stereotactic biopsy in $91 \%$ of all cases, with a major morbidity rate of $3.5 \%$ and an operative mortality rate of only $0.7 \% .^{[27]}$ Dorward et $a l^{[4]}$ compared frameless and frame-based biopsy techniques and found superior imaging, target visualization and flexibility of the frameless stereotactic biopsy when compared with the current gold-standard frame-based biopsy. Recently, Spivak and Pirouzmand also proved that image-guided stereotactic neuronavigational systems are more accurate. ${ }^{[28]}$ Results of the present study are comparable as diagnostic yield was maximum in image-guided procedure, which may reflect the ability to perform interactive, intraoperative scanning with this technique. On the other hand, Smith et al found that frameless image-guided and frame-based stereotactic approaches are equally effective in terms of tissue diagnosis. ${ }^{[29]}$ They found that imageguided surgical navigation system is less cost-effective and requires long hospital stay.

Therefore, to overcome the disadvantages related to stereotactic procedure, in terms of cost of the stereotactic equipment and time length of procedure, the search for an alternative technique has focused on ultrasound-guided brain biopsy. In comparison to these methods, this procedure is quick, less expensive and requires less scanning. ${ }^{[30]}$ The development of the B-mode real time ultrasound technique has furnished a simpler method of visualizing intracranial lesions. These technological advances are largely due to the efforts of Berger and Tsutsumi, ${ }^{[6,31]}$ who created surgical sets for ultrasound-guided biopsy. The Berger neuro biopsy device is considerably cheaper than all stereotactic frames and saves anesthetic and CT time. However, the ultrasound-guided biopsy is currently indicated for supratentorial lesions exceeding $5 \mathrm{~mm}$ in diameter which do not require a milimetric spatial discrimination. ${ }^{[6]}$ Fujita et al compared various image-guided brain biopsy procedures and concluded that the US-guided procedure is suitable for the investigation of hyperdense lesions, but it is difficult to localize nonechogenic lesions. However, the combination of ultrasound and neuronavigator methods improved the diagnostic accuracy even in nonechogenic lesions. ${ }^{[32]}$ Lorenzo 
et $a l^{[33]}$ compared the diagnostic accuracy of $\mathrm{CT}$ and US-guided stereotactic brain biopsies and concluded that these two procedures are complementary. Although diagnostic yield was more in the CT-guided stereotactic group than in the US-guided group of patients. Similar results were noted in the present series.

In the present study, maximum diagnostic yield was achieved with frameless image-guided stereotactic biopsy followed by framebased CT-guided stereotaxis and US-guided biopsy. The results indicate that the visually controlled real-time procedure gives rise to accurate target determination and finally to greatest increase in diagnostic yield. Image-guided biopsies were found to be better in terms of diagnostic yield than frame-based stereotaxis and the US-guided group in the present study. However, it is not statistically significant ( $P$ value 0.85 ) in view of the small number of cases included in the frameless and US-guided biopsy groups.

\section{Conclusion}

Therefore it is concluded that the advantage of near real-time intraoperative imaging may include high yield of pathological tissue that is essential for making therapeutic decisions. The high percentage of satisfactory neuropathological diagnoses, with the assistance of computer and stereotactic neuronavigational system justify this kind of diagnostic measures as a routine method.

\section{References}

1. Apuzzo ML, Sabshin JK. Computed tomography guidance stereotaxis in the management of intracranial mass lesions. Neurosurgery 1983;12:277-85.

2. Speigal EA, Wyeis HT, Marks M, Jee A.J. Stereotaxic apparatus for operations on the human brain. Science 1947;106:349-50.

3. Leksell L. A stereotaxic apparatus for intracerebral surgery. Acta Chir Scand 1949;99:229-33

4. Dorward NL, Paleologos TS, Alberti O, Thomas DG. The advantages of frameless stereotactic biopsy over frame-based biopsy. Br J Neurosurg 2002;16:110-8.

5. Matsumoto K, Tomita S, Higashi H, Nakagawa M, Adachi H, Tada E, et al. Image guided stereotactic biopsy for brain tumors: Experience of 71 cases. No Shinkei Geka 1995;23:897-903.

6. Berger MS. Ultrasound guided stereotaxic biopsy using a new apparatus. J Neurosurg 1986;65:550-64.

7. Enzmann DR, Irvin KM, Marshall WH, Silverberg GD, Britt RH, Hanbery JW. Intraoperative sonography through a burr hole: Guide for brain biopsy. A.JNR Am J Neuroradiol 1984;5:243-6.

8. Kelly PJ. Computer assisted stereotaxis: New approaches for the management of intracranial intra-axial tumors. Neurology 1986;36:535-41.

9. Broggi G, Franzini A, Giorgi C, Allegranza A. Diagnostic accuracy of multimodel approach in stereotactic biopsies of deep brain tumors. Acta Neurochirurgica (Suppl) 1984;33:211-2.

10. Hayden R, Cajulis RS, Frias-Hidvegi D, Brody BA, Yu G, Levy R. Intraoperative diagnostic techniques for stereotactic brain biopsy: Cytology versus frozen-section histopathology. Stereotact Funct Neurosurg 1995;65:187-93.

11. Kitchen ND, Bradford R, McLaughlin JE. The value of per operative smear examination during stereotactic biopsy. Acta Neurochir (Wien) 1993;121:196-8.

12. Liwnicz BH, Henderson KS, Masukawa T, Smith RD. Needle aspiration cytology of intracranial lesions. A review of 84 cases. Acta Cytol 1982;26:779-86.

13. Marshall LF, Adams H, Doyle D, Graham DI. The histological accuracy of the smear technique for neurosurgical biopsies. J Neurosurg 1973;39:82-8.

14. Martinez A.J, Pollack I, Hall WA, Lunsford LD. Touch preparations in the rapid intraoperative diagnosis of central nervous system lesions. A comparison with frozen sections and paraffin-embedded sections. Mod Pathol 1988;1:378-84.

15. Firlik KS, Martinez A.J, Lunsford LD. Use of cytological preparations for the intraoperative diagnosis of stereotactically obtained brain biopsies: A 19-year experience and survey of neuropathologists. J Neurosurg 1999;91:454-8.

16. Collaco LM, Tani E, Lindblom I, Skoog L. Stereotactic biopsy and cytological diagnosis of solid and cystic intracranial lesions. Cytopathology 2003;14:131-5.

17. Grunert P, Ungersbock K, Bohl J, Kitz K, Hopf N. Results of 200 intracranial stereotactic biopsies. Neurosurg Rev 1994;17:59-66.

18. Chandrasoma PT, Smith MM, Apuzzo ML. Stereotactic biopsy in the diagnosis of brain masses: Comparison of results of biopsy and resected surgical specimen. Neurosurgery 1989;24:160-5.

19. Bernays RL, Kollias SS, Khan N, Brandner S, Meier S, Yonekawa Y. Histological yield, complications and technological considerations in 114 consecutive frameless stereotactic biopsy procedures aided by open intraoperative magnetic resonance imaging. J Neurosurg 2002;97:354-62.

20. McGirt M.J, Villavicencio AT, Bulsara KR, Friedman AH. MRI-guided stereotactic biopsy in the diagnosis of glioma: Comparison of biopsy and surgical resection specimen. Surg Neurol 2003;59:277-82.

21. Aker FV, Hakan T, Karadereler S, Erkan M. Accuracy and diagnostic yield of stereotactic biopsy in the diagnosis of brain masses: Comparison of results of biopsy and resected surgical specimens. Neuropathology 2005;25:207-13.

22. Kepes JJ. Pitfalls and problems in the histopathologic evaluation of stereotactic needle biopsy specimens. Neurosurg Clin N Am 1994;5:19-33.

23. Goldstein S, Gumerlock MK, Neuwelt EA. Comparison of CT guided and stereotaxic cranial diagnostic needle biopsies. J Neurosurg 1987;67:341-8.

24. Henderson JM. Frameless localization for functional neurosurgical procedures: A preliminary aceuracy study. Stereotact Funct Neurosurg 2004;82:135-41.

25. Moriarty TM, Quinones-Hinojosa A, Larson PS, Alexander E $3^{\text {rd }}$, Gleason PL, Schwartz RB, et al. Frameless stereotactic neurosurgery using intraoperative magnetic resonance imaging: Stereotactic brain biopsy. Neurosurgery 2000;47:1138-46

26. MeGirt MJ, Woodworth GF, Coon AL, Frazier JM, Amundson E, Garonzik I, et al Independent predictors of morbidity after image guided stereotactic brain biopsy: A risk assessment of 270 cases. J Neurosurg 2005; 102:897-901.

Gralla J, Nimsky C, Buchfelder M, Fahlbusch R, Ganslandt O. Frameless stereotactic brain biopsy procedures using the stealth station: Indications, accuracy and results. Zentralbl Neurochir 2003;64:166-70.

28. Spivak C.J, Pirouzmand F. Comparison of the reliability of brain lesion localization when using traditional and stereotactic image-guided techniques: A prospective study. J Neurosurg 2005;103:424-7.

29. Smith JS, Quinones-Hinojosa A, Barbaro NM, McDermott MW. Frame-based stereotactic biopsy remains an important diagnostic tool with distinct advantages over frameless stereotactic biopsy. J Neurooncol 2005;73:173-9.

30. Lunardi P, Acqui M, Maleci A, Lorenzo ND, Fortuna A. Ultrasound guided brain biopsy: A personal experience with emphasis on its indication. Surg Neurol $1993 ; 39: 148-51$

31. Tsutsumi Y, Andoh Y, Sakaguchi .J. A new ultrasound guided brain biopsy technique through a burr hole. Technical note. Acta Neurochir (Wien) 1989;96:72-5.

32. Fujita K, Yanaka K, Meguro K, Narushima K, Iguchi M, Nakai Y, et al. Image guided procedures in brain biopsy. Neuro Med Chir (Tokyo) 1999;39:502-9.

33. Di Lorenzo N, Esposito V, Lunardi P, Delfini R, Fortuna A, Cantore G. A comparison of computerized tomography guided stereotactic and ultrasound guided techniques for brain biopsy. J Neurosurg 1991;75:763-5.

Accepted on 31-08-2006

Source of Support: Nil, Conflict of Interest: None declared. 